\title{
Lights and shadows of schizophrenia therapy research: lessons from oral risperidone and olanzapine
}

\section{Supplemental Material}

SM1. Overtime distribution of the number of individuals enrolled in the selected primary studies (PSs).

The total number of patients enrolled in PSs each year were annotated and analyzed, in order to verify if the pattern of PS published articles (see Fig. 2c in the main article) also reflected the overtime distribution of the PS patient population receiving oral risperidone (oRISP) or oral olanzapine (oOLA) conventional tablets.

The time-dependent distribution of the total number of individuals enrolled in PSs showed a rapid decline of new empirical data on oRISP+oOLA in the recent years (Fig. 1S). It should be noted that two clinical studies (Strom et al., 2011; Thomas et al., 2010) were not included in the regression curve analysis. These exceptionally large clinical studies focusing on the safety of sertindole and ziprasidone did not appear to be statistically representative of the oRISP+oOLA research pattern, since they 
accomplish Tukey's fences criterion for "far out outliers", i.e. $x \geq Q 3+3(Q 3-Q 1)$ (Cohen et al., 2003; Draper and Smith, 1998).

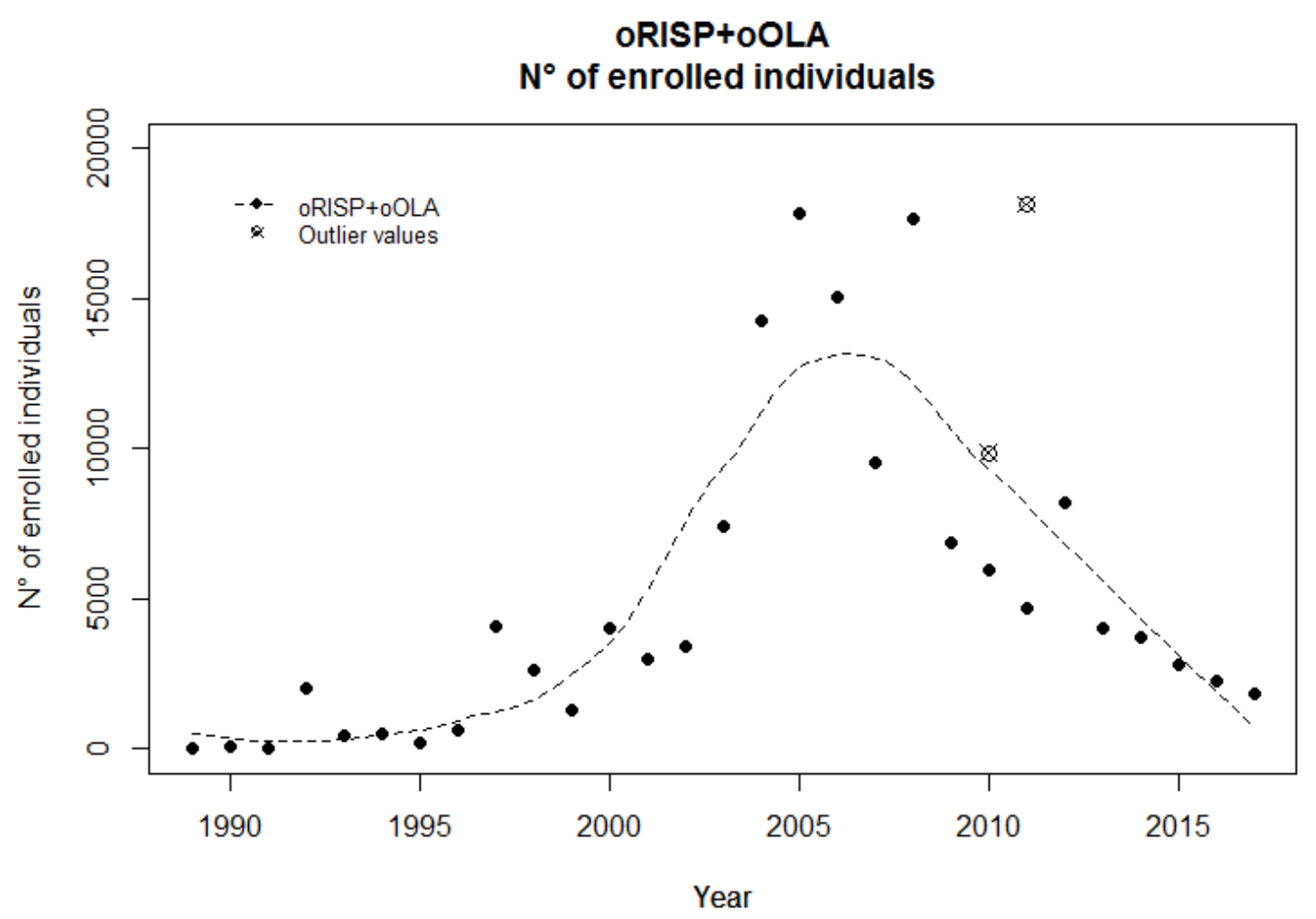

Fig. 1S - The graph depicts the time-dependent distribution of the total number of individuals enrolled in published primary studies (PSs) which investigated the clinical properties of oRISP+oOLA (filled circles $(\bullet)$ symbol).

Crossed circle $(\otimes)$ symbol shows the outlier values not included in the regression curve analysis. 
SM2. Comparison of the downward trends shown by industrial supported (IS) vs. independent research (IndR) studies in the last 9 years (2009-2017).

Simple linear regression models of the number of published research articles investigating the clinical properties of oRISP+oOLA were used in order to highlight possible differences in the downward pattern shown by IS and IndR published articles over the last 9 years (2009-2017). With this purpose in mind, Shapiro-Wilk normality test (alpha level $=0.05)$ was applied in order to verify whether IS and IndR data (20092017) came from normally distributed populations (Rahman and Govidarajulu, 1997; Shapiro and Wilk, 1965). Then, the statistical difference between the linear regressions coming from IndR vs. IS data was analyzed using two statistical approaches. Firstly, a two-tail t-Test (alpha level $=0.05$ ) was applied in order to compare a possible statistical difference between the linear regression slopes of the two groups. Moreover, the reciprocal time-dependent distribution of the number of IndR vs. IS published articles was analyzed using point to point comparisons of predicted values of the two model solutions (Cohen et al., 2003; Draper and Smith, 1998).

The statistical analyses indicated that the linear regression slopes of both IndR and IS were significantly different from 0 (IndR: $b=-1.3833$, S.E. $=0.3803, t=-3.638, p<$ 0.01 ; IS: $b=-4.4500$, S.E. $=0.6359, t=-6.998, p<0.001$ ) (Fig. $2 S$ ), when the number of articles published between 2009 and 2017 were considered. Shapiro analyses 
confirmed the normality of IndR and IS data distribution (IndR: $W=0.88524, p=0.1781$; IS: $W=0.87891, p=0.1529)$. A significant difference was found when the linear regression slopes of IndR and IS were compared using a t-test procedure $(t=-8.911$, p<0.001) (Fig. 2S). This finding was consistent with the results of the point-to-point analysis of the predicted values, remarking the crossover of the two regression lines (Fig. 2S). Indeed, the number of IndR articles was lower than that of IS when analyzed between 2009 and 2011 ( $p<0.05$ ). Subsequently, a comparable number of IndR and IS articles could be observed during 2012 and 2013 ( $p>0.05$ ), while the number of IndR articles became higher than that of IS after $2014(p<0.05)$. 
IndR vs. IS

(2009-2017)

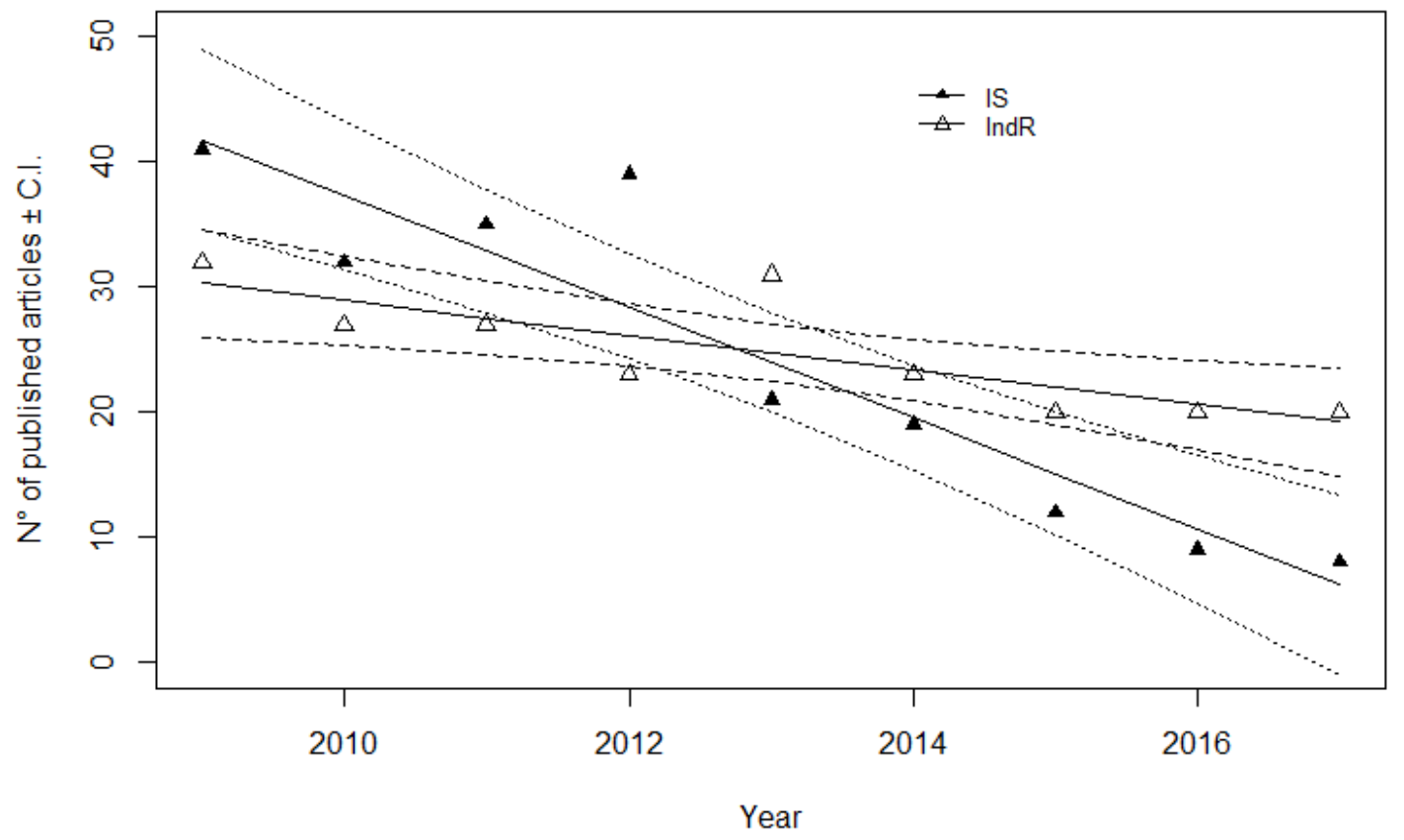

Fig. $2 S$ - The graph depicts the linear regression slopes \pm C.I. of the number of IndR and IS articles which had been published between 2009 and 2017. 


\section{Abbreviation list}

\section{Table 1S}

\begin{tabular}{ll} 
Abbreviation & Definition \\
OOLA & Oral olanzapine \\
oRISP & Oral risperidone \\
PSS & Primary studies \\
SAs & Secondary/adjunctive analyses \\
IS & Industrial supported \\
IndR & Independent research \\
ND & Not declared \\
MC & Manufacturer/selling company \\
CC & Competitor company \\
OthCs & Other competitors \\
\hline \hline
\end{tabular}




\section{References of Supplemental Material}

Cohen J, Cohen P, West SG, and al. (2003) Applied multiple regression-correlation analysis for the behavioral sciences. $3^{\text {rd }}$ Ed Mahwah, London, UK, Erlbaum.

Draper NR and Smith H (1998) Applied regression analysis. $3^{\text {rd }}$ Ed, New York, NY, Wiley.

Rahman MM and Govidarajulu Z (1997) A modification of the test of Shapiro and Wilk for normality. J App/ Stat 24(2): 219-236.

Shapiro SS and Wilk MB (1965) An analysis of variance test for normality (complete samples). Biometrika, 52: pp 591-611.

Strom BL, Eng SM, Faich G, et al. (2011) Comparative mortality associated with ziprasidone and olanzapine in real-world use among 18,154 patients with schizophrenia: The Ziprasidone Observational Study of Cardiac Outcomes (ZODIAC). Am J Psychiatry 168(2): 193-201.

Thomas SH, Drici MD, Hall GC, et al. (2010) Safety of sertindole versus risperidone in schizophrenia: principal results of the sertindole cohort prospective study (SCoP). Acta Psychiatr Scand 122(5): 345-355. 